\title{
Efeito cariostático de restaurações adesivas em superfícies radiculares: estudo in vitro
}

\section{Caries inhibition around adhesive restorations in roots: in vitro study}

\author{
Anderson Takeo HARA* \\ Cláudia Silami de MAGALHÃES** \\ Antonio Luiz RODRIGUES Jr.*** \\ Mônica Campos SERRA****
}

\begin{abstract}
HARA, A. T.; MAGALHÃES, C. S. de; RODRIGUES Jr., A. L.; SERRA, M. C. Efeito cariostático de restaurações adesivas em superficies radiculares: estudo in vitro. Pesq Odont Bras, v. 14, n. 2, p. 113-118, abr./jun. 2000.

Materiais restauradores que liberam íons flúor e/ou promovem adesão à estrutura dental têm sido relacionados com a inibição do desenvolvimento de lesões de cárie adjacentes às restaurações. A hipótese testada neste estudo foi a de que o uso de resina composta/sistema adesivo tem efeito cariostático semelhante a um material adesivo que libera íons flúor - cimento de ionômero de vidro - sobre a superfície radicular adjacente às restaurações. Foram utilizadas 20 raízes de terceiros molares humanos extraídos, embutidas em resina de poliestireno e planificadas. Cavidades padronizadas foram preparadas e restauradas aleatoriamente com (a) Chelon-Fil (Espe) ou (b) Z100/SingleBond (3M). Valores iniciais $\left(K H N_{i}\right)$ de microdureza superficial Knoop da dentina foram obtidos a 100, 200 e $300 \mu \mathrm{m}$ da margem oclusal das restaurações. Uma área de $2,0 \mathrm{~mm}$ ao redor da restauração foi delimitada e submetida à indução de cárie artificial. Obtiveram-se, então, os valores finais $\left(K H N_{f}\right)$ de microdureza, nas mesmas condições e localizações da leitura inicial. As diferenças entre $K H N_{i}$ e $K H N_{f}$ foram consideradas para a análise estatística. As medianas de $K H N_{i}-K H N_{f}$ nas distâncias de 100, 200 e $300 \mu \mathrm{m}$ foram para (a): -3,8; -0,3; -1,0; e para (b): 3,3; 2,5; 1,7. O teste de Kruskal-Wallis não evidenciou diferença significativa entre as distâncias dentro de cada grupo. Às distâncias de 200 e $300 \mu \mathrm{m}$, não houve diferença significativa entre os materiais avaliados. À distância de $100 \mu \mathrm{m}$, (a) diferiu significativamente de (b) $(p<0,05)$. Sob as condições deste estudo, o cimento de ionômero de vidro apresentou maior potencial cariostático que a resina composta com sistema adesivo dentinário.
\end{abstract}

UNITERMOS: Cárie radicular; Cariostáticos; Materiais dentários.

\section{INTRODUÇÃO}

A formação e o desenvolvimento da cárie radicular são semelhantes aos da lesão coronária, necessitando da presença de raiz dental exposta ao ambiente oral, da deficiência no controle de placa bacteriana - formada por uma microbiota específica - e da adoção de uma dieta cariogênica ${ }^{32}$.

O conhecimento desses fatores etiológicos permite intervir no desenvolvimento do processo carioso, através de medidas de promoção de saúde, possibilitando a sua paralisação ou reversão $0^{10,20,21}$. No entanto, em situações onde a profundidade da lesão impedir uma correta higienização, onde houver grande sensibilidade pulpar, necessidade do restabelecimento da forma e funções dentais, ou ainda em locais de envolvimento estético, o tratamento restaurador se faz necessário ${ }^{6,10}$.

A técnica restauradora de lesões cariosas radiculares pode apresentar vários inconvenientes, dentre os quais destacam-se a dificuldade no isolamento do campo operatório ${ }^{2}$, o dificil acesso à le$\mathrm{são}^{23} \mathrm{e}$ a inexistência de um material que propicie uma ótima adesão à dentina. Conseqüentemente, esses fatores podem contribuir para o insucesso do tratamento, principalmente pela ocorrência de cárie radicular adjacente à restauração ${ }^{15}$. A formação e progressão dessa lesão, embora questionada $^{31}$, tem sido atribuída à presença de duas frentes de desmineralização, uma na superfície anatômica do dente e outra na interface dente-material restaurador ${ }^{3,15}$.

\footnotetext{
*Aluno de Mestrado em Clínica Odontológica; **** Professora Livre-Docente na área de Dentística - Faculdade de Odontologia de Piracicaba da UNICAMP.

** Professora Assistente do Departamento de Odontologia Restauradora - Faculdade de Odontologia da UFMG.

*** Professor Assistente nas áreas de Bioestatística e Metodologia Científica - Faculdade de Odontologia de Araraquara da UNESP.
} 
HARA, A. T.; MAGALHÃES, C. S. de; RODRIGUES Jr., A. L.; SERRA, M. C. Efeito cariostático de restaurações adesivas em superficies radiculares: estudo in vitro. Pesq Odont Bras, v. 14, n. 2, p. 113-118, abr./jun. 2000.

Supõe-se que o desenvolvimento das resinas compostas e dos sistemas adesivos hidrófilos venha minimizar a recorrência de cárie ao redor da restauração. Avaliações laboratoriais desses materiais têm demonstrado baixos índices de microinfiltração em substrato dentinário ${ }^{1,28}$, contribuindo, hipoteticamente, para a redução dos efeitos de desmineralização radicular junto à interface dente-material restaurador. No entanto, a efetividade e a amplitude desse efeito preventivo frente ao ataque cariogênico são ainda desconhecidas.

Os cimentos de ionômero de vidro, desenvolvidos no início da década de $70^{33}$, também têm sido relacionados à inibição da recorrência de lesões cariosas $^{24}$. Esse material apresenta como característica principal a liberação de íons flúor ${ }^{2,11}$, os quais, dentro da dinâmica envolvida no desenvolvimento da cárie, podem apresentar um efeito antimicrobiano, inibir a desmineralização e potencializar a remineralização dental ${ }^{7}$.

Embora o potencial cariostático de materiais que liberam íons flúor seja bem documentado na literatura, tanto em esmalte ${ }^{26,27}$, como em dentina radicular ${ }^{4,14,16,29}$, faz-se necessário uma comparação específica entre esse recurso de prevenção da formação e progressão da cárie adjacente à restauração e outro baseado na eficiência do vedamento da interface dente-restauração.

\section{PROPOSIÇÃO}

Este estudo in vitro teve por objetivo comparar a eficácia de dois materiais restauradores - um ionômero de vidro convencional e uma resina composta/sistema adesivo hidrófilo não fluoretados - na inibição do desenvolvimento de lesões de cárie radicular artificial, adjacentes às restaurações.

\section{MATERIAL E MÉTODO Preparo dos corpos-de-prova}

Foram utilizados fragmentos dentais provenientes de 20 terceiros molares humanos, extraídos por motivos não relacionados à execução deste estudo. Após as extrações, os dentes foram limpos com curetas periodontais, polidos com pasta de pedra-pomes e água, utilizando taça de borracha em baixa rotação, e mantidos em solução tamponada de formol a $10 \%$.

Seccionou-se - com disco diamantado dupla face (ref. 070, KG Sorensen) em baixa rotação - a região cervical de cada dente, de modo a separar a coroa da raiz. Desprezou-se a parte coronária. Novas secções foram feitas na porção radicular, obtendo-se um fragmento de aproximadamente $5,0 \mathrm{~mm}$ de largura por 5,0 $\mathrm{mm}$ de comprimento e
2,0 mm de espessura. Uma demarcação foi realizada na porção oclusal do fragmento, de maneira a facilitar a sua identificação no teste de microdureza. Os fragmentos foram separados, aleatoriamente, em dois grupos de 10 unidades cada.

Os fragmentos foram incluídos, individualmente, em 20 blocos de resina de poliestireno (Ortoftálica Cristal, Cromex). Após a inclusão, a superfície radicular dos corpos-de-prova apresentou-se exposta, para subseqüente aplainamento e polimento em politriz giratória (Maxigrind, Solotest), refrigerada a água, com lixas de óxido de alumínio de granulações 600 e 1.000 (Carborundun Abrasivos). Após esse procedimento, a superficie dental apresentou-se plana e com, no mínimo, uma área circular de 4,0 mm de diâmetro.

Foram realizadas cavidades de aproximadamente 2,0 $\mathrm{mm}$ de diâmetro por 1,0 $\mathrm{mm}$ de profundidade, na superficie de cada fragmento, utilizando pontas diamantadas cilíndricas (KG Sorensen), em alta rotação, refrigeradas por jato de ar/água. As cavidades foram então restauradas, aleatoriamente, conforme o material indicado para cada grupo (Quadro 1), seguindo-se as instruções fornecidas por seus respectivos fabricantes.

Os corpos-de-prova foram, então, armazenados separadamente de acordo com cada grupo, em ambiente úmido, numa estufa a $37^{\circ} \mathrm{C}( \pm 1)$, por 24 horas. Em seguida, as restaurações foram submetidas ao acabamento, com lixas de óxido de alumínio de granulação 1.000 (Carborundun Abrasivos), em politriz giratória refrigerada a água. A fim de determinar a área a ser submetida à indução de cárie, os fragmentos foram isolados com esmalte de unha (Risqué, Niasi S.A.), à exceção da restauração e da superfície radicular localizada a 1,0 $\mathrm{mm}$ de diâmetro ao redor da mesma.

\section{Indução de cárie}

A indução de cárie artificial foi realizada por um modelo dinâmico de desmineralização e remineralização, simulando condições in vivo de alto risco de cárie, semelhante ao proposto por FEATHERSTONE $^{8}$ (1986) e modificado por SERRA; CURY ${ }^{26}$

QUADRO 1 - Grupos, tipos e fabricantes dos materiais restauradores utilizados.

\begin{tabular}{c|l|l}
\hline \hline \multicolumn{1}{c|}{ Grupos } & \multicolumn{1}{|c|}{ Tipo de Material } & \multicolumn{1}{c}{ Fabricante } \\
\hline 1. Chelon-Fil & $\begin{array}{l}\text { Cimento de } \\
\text { ionômero de vidro }\end{array}$ & $\begin{array}{l}\text { Espe GmbH } \\
\text { Seefeld, Germany }\end{array}$ \\
\hline $\begin{array}{c}\text { 2. Z100 / Sin- } \\
\text { gle Bond }\end{array}$ & $\begin{array}{l}\text { Resina composta } \\
\text { microíbrida / Sistema } \\
\text { adesivo hidrófilo }\end{array}$ & $\begin{array}{l}\text { 3M Dental Products } \\
\text { St. Paul, MN, USA }\end{array}$ \\
\hline \hline
\end{tabular}


HARA, A. T.; MAGALHÃES, C. S. de; RODRIGUES Jr., A. L.; SERRA, M. C. Efeito cariostático de restaurações adesivas em superfícies radiculares: estudo in vitro. Pesq Odont Bras, v. 14, n. 2, p. 113-118, abr./jun. 2000.

(1992). Os corpos-de-prova foram submetidos a três ciclos de desmineralização e remineralização, separados de acordo com os grupos de materiais. Cada ciclo consistiu na imersão dos corpos-deprova em solução remineralizante $(\mathrm{pH} 7,0)$ por 23 horas e em solução desmineralizante ( $\mathrm{pH} 4,3)$, por 1 hora. Entre as trocas de soluções os corposde-prova foram abundantemente lavados com água destilada e deionizada. Em seguida, foram armazenados individualmente, imersos em água destilada e deionizada, em estufa a $37^{\circ} \mathrm{C}( \pm 1)$.

\section{Avaliação da microdureza}

O desenvolvimento das lesões de cárie artificial foi quantificado por meio de ensaios de microdureza Knoop na superficie dentinária adjacente à restauração. Os valores de dureza foram obtidos an-

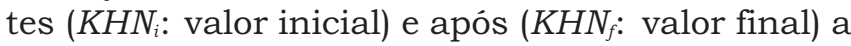
indução de cárie artificial, utilizando microdurômetro (FM 1E, Future Tech. Co.), com diamante Knoop e carga estática de $10 \mathrm{~g}$, aplicada por $15 \mathrm{~s}$. Foram realizadas três endentações adjacentes à restauração, posicionando-se o longo eixo do diamante paralelamente à superficie dentinária. As endentações - paralelas entre si - localizaram-se na parte oclusal de cada fragmento a 100, 200 e $300 \mathrm{~mm}$ da restauração.

Os valores de microdureza foram obtidos através da equação:

$$
K H N=\frac{14229 \cdot P}{l^{2}}
$$

Onde KHN é o número de dureza Knoop, $P$ é a força aplicada, em gramas, e $l$ é o comprimento da endentação em micrômetros. Foram consideradas para a análise estatística as diferenças encontradas entre os valores iniciais e valores finais de microdureza $\left(K H N_{\mathrm{i}}-K H N_{\mathrm{f}}\right)$.

\section{Análise estatística}

Os valores de microdureza Knoop $\left(K H N_{\mathrm{i}}-K H N_{\mathrm{f}}\right)$ foram analisados estatisticamente pelo método não-paramétrico de Kruskal-Wallis, considerando um nível de significância de 5\%, para avaliação do efeito dos fatores tratamento restaurador e distância da endentação à restauração.

\section{RESULTADOS}

A Tabela 1 expressa as medianas das diferenças obtidas para cada grupo, dentro das distâncias avaliadas. O teste de Kruskal-Wallis evidenciou uma diferença significativa entre os tratamentos restauradores, na distância de $100 \mu \mathrm{m}$ da restauração $(p=0,03)$. Nas distâncias de 200 e $300 \mu \mathrm{m}$ não foram observadas diferenças significativas entre os dois materiais, com $p=0,12$ e $p=0,21$, respectivamente (Tabela 1).

Nas avaliações entre as distâncias - 100, 200 e $300 \mu \mathrm{m}$ - não foram observadas diferenças estatisticamente significativas para o cimento de ionômero de vidro $(p=0,63)$ assim como para a resina composta $(\mathrm{p}=0,60)($ Tabelas 2 e 3$)$.

\section{DISCUSSÃO}

Estudos da liberação de íons flúor ${ }^{11,17}$ bem como o da microinfiltração de materiais restauradores $^{1,25,28}$ têm sido realizados e relacionados com a sua capacidade de prevenir o desenvolvimento de cárie adjacente às restaurações. No entanto, quando realizadas individualmente, tais avaliações são pouco capazes de predizer o potencial cariostático dos materiais estudados ${ }^{5,16}$. Para obter resultados mais próximos da realidade, pode ser indicada a realização de testes laboratoriais, envolvendo a formação de cárie artificial adjacente às restaurações, considerando, simultaneamente, as influências da microinfiltração e da liberação de íons flúor pelo material ${ }^{29}$.

Modelos de indução de cárie artificial em raiz são relatados na literatura, destacando-se os de desmineralização ${ }^{23,29}$ e os de ciclagens de des-remineralização ${ }^{2}$. De acordo com a dinâmica envolvida no processo de formação e progressão da cárie, a utilização de ciclos alternados des-remineralizantes parece melhor simular as condições in vivo de

TABELA 1 - Mediana das diferenças entre os valores de microdureza $\left(K H N_{i}-K H N_{f}\right)$ e análise entre os materiais dentro de cada distância.

\begin{tabular}{l|c|c|c|c|c|c}
\hline \hline \multirow{2}{*}{ Grupos } & \multicolumn{6}{|c}{ Distâncias $(\mathrm{m})$} \\
\cline { 2 - 7 } & \multicolumn{2}{|c|}{$100(\mathrm{p}=0,03)$} & \multicolumn{2}{c}{$200(\mathrm{p}=0,12)$} & \multicolumn{2}{c}{$300(\mathrm{p}=0,21)$} \\
\cline { 2 - 7 } & Mediana & Postos médios & Mediana & Postos médios & Mediana & Postos médios \\
\hline Resina composta & 3,3 & $12,94 \mathrm{a}$ & 2,5 & $12,11 \mathrm{a}$ & 1,7 & $11,67 \mathrm{a}$ \\
\hline Ionômero de vidro & 3,8 & $7,35 \mathrm{~b}$ & 0,3 & $8,10 \mathrm{a}$ & 1,0 & $8,50 \mathrm{a}$ \\
\hline \hline
\end{tabular}

Letras diferentes indicam diferença significativa entre materiais $(p<0,05)$, dentro de cada distância. 
HARA, A. T.; MAGALHÃES, C. S. de; RODRIGUES Jr., A. L.; SERRA, M. C. Efeito cariostático de restaurações adesivas em superficies radiculares: estudo in vitro. Pesq Odont Bras, v. 14, n. 2, p. 113-118, abr./jun. 2000.

TABELA 2 - Análise entre distâncias do grupo resina composta.

\begin{tabular}{c|c|c}
\hline \hline $\begin{array}{c}\text { Resina composta } \\
(\text { distância em } \mu \mathrm{m})\end{array}$ & Mediana & Postos médios \\
\hline 100 & 3,3 & $16,00 \mathrm{a}$ \\
\hline 200 & 2,5 & $13,78 \mathrm{a}$ \\
\hline 300 & 1,7 & $12,22 \mathrm{a}$ \\
\hline \hline
\end{tabular}

Letras iguais indicam não haver diferenças significativas $(\mathrm{p}=0,60)$.

alto risco a essa lesão ${ }^{8,30}$. Porém, a aplicação desses modelos em dentina radicular induz a formação de erosões superficiais, o que limita o uso do ensaio de microdureza para avaliar lesões de subsuperficie, em cortes longitudinais através da restauração/lesão. Avaliações por meio de microrradiografias $^{2,19}$ e por microscopia de luz polarizada ${ }^{3,23}$ não têm sido prejudicadas pela ocorrência de erosões. Entretanto, ao contrário da microdureza, a análise por microscopia de luz polarizada não quantifica a perda mineral dental, e a por microrradiografia não considera o conteúdo orgânico do dente ${ }^{26}$.

Assim, para viabilizar o estudo da microdureza de lesões radiculares adjacentes a restaurações utilizando um modelo de indução de cárie em esmalte com ciclagem de $\mathrm{pH}^{8}$ - propôs-se, neste estudo, uma redução do número de ciclos de des-remineralização, bem como do tempo de imersão de cada corpo-de-prova em solução desmineralizante. Outro recurso aplicado foi a leitura de microdureza na superficie dentinária, antes e depois da formação da lesão, numa mesma região - oclusal ao redor da restauração. Esse fato possibilitou um maior controle das variáveis relacionadas a cada corpo-de-prova, uma vez que foram consideradas, para análise estatística, as diferenças entre os valores iniciais e finais $\left(K H N_{\mathrm{i}}-K H N_{\mathrm{f}}\right)$ de microdureza de cada corpo-de-prova.

A significativa diferença encontrada entre os dois materiais avaliados, na distância de $100 \mu \mathrm{m}$ da restauração, demonstra um possivel efeito cariostático do cimento de ionômero de vidro convencional, ao contrário do compósito avaliado. Esse resultado está de acordo com outros observados em avaliações anteriores ${ }^{14,23}$, comprovando a efetividade do material ionomérico na prevenção da cárie radicular, dentro das condições adotadas na realização deste tipo de estudo. O suposto efeito benéfico advindo da melhor adesão entre o sistema adesivo/resina composta e estrutura dental não foi observado.
TABELA 3 - Análise entre distâncias do grupo ionômero de vidro.

\begin{tabular}{c|c|c}
\hline \hline $\begin{array}{c}\text { Ionômero de vidro } \\
(\text { distância em } \mu \mathrm{m})\end{array}$ & Mediana & Postos médios \\
\hline 100 & $-3,8$ & $13,40 \mathrm{a}$ \\
\hline 200 & $-0,3$ & $16,05 \mathrm{a}$ \\
\hline 300 & $-1,0$ & $17,05 \mathrm{a}$ \\
\hline \hline
\end{tabular}

Letras iguais indicam não haver diferenças significativas $(p=0,63)$.

Embora a avaliação de lesões de parede - originárias da interface dente-material restaurador não tenha sido objeto de estudo desta pesquisa, sugere-se, com base em outros trabalhos ${ }^{19,23,29}$, que o uso de uma resina composta associada ao sistema adesivo não tenha sido suficiente para inibir a formação e progressão da cárie artificial adjacente à restauração, como foi observado ao se utilizar o cimento ionomérico. Dessa forma é possivel supor, dentro das condições laboratoriais adotadas, que um bom vedamento entre dente e restauração não seja suficiente para inibir cárie, se o material em questão não apresentar liberação de quantidades adequadas de flúor nas formas iônica ou ionizável.

Ainda que avaliações laboratoriais tenham demonstrado menores índices de microinfiltração com o uso dos sistemas adesivos hidrófilos ${ }^{1,25}$, o seu total controle, principalmente em substrato dentinário, ainda não foi relatado. Neste estudo, a microinfiltração pode ter pronunciado a progressão da lesão cariosa. Porém, avaliações futuras são necessárias para predizer se a ausência da microinfiltração acarretaria maiores valores de microdureza à dentina adjacente às restaurações de resina composta/sistema adesivo, indicando, assim, uma inibição ou controle do desenvolvimento de cáries adjacentes às mesmas.

Alguns autores ${ }^{31}$ têm questionado, baseados em experiência clínica, a real influência da lesão de parede na cárie adjacente à restauração. Ou seja, a ocorrência de fendas visíveis, discrepância marginal e microinfiltração estaria pouco relacionada com esse tipo de lesão cariosa, sendo a mesma resultante de uma série de condições locais que propiciem o desenvolvimento de uma placa bacteriana cariogênica $^{31}$. Essa observação clínica ressalta, num primeiro aspecto, a importância da utilização de medidas de promoção de saúde, como o adequado controle da placa bacteriana; e, além disso, a importância de outras medidas complementares, 
HARA, A. T.; MAGALHÃES, C. S. de; RODRIGUES Jr., A. L.; SERRA, M. C. Efeito cariostático de restaurações adesivas em superfícies radiculares: estudo in vitro. Pesq Odont Bras, v. 14, n. 2, p. 113-118, abr./jun. 2000.

como a utilização das várias fontes de fluoretos, dentre as quais o material restaurador.

Estudos de longevidade têm apontado como a principal causa de fracasso das restaurações de resina composta a recorrência de cárie ${ }^{18}$, e das restaurações de cimento de ionômero de vidro, a incidência de fraturas ${ }^{18,22}$. O conhecimento das limitações desses materiais permite especificar suas indicações de acordo com cada situação clínica. Visando ao melhor desempenho clínico das restaurações, foram desenvolvidos materiais híbridos de ionômero de vidro/resina composta, pela incorporação de componentes resinosos ao cimento de ionômero de vidro convencional. Espera-se, assim, um somatório das qualidades dos dois materiais que os originaram ${ }^{17}$. Surgiram, ainda, as resinas compostas e sistemas adesivos contendo fluoretos. Novas avaliações são necessárias para determinar a influência desses materiais frente ao desenvolvimento da cárie radicular.

Em estudos laboratoriais, diversas condições comuns ao ambiente bucal são desprezadas com o objetivo de evidenciar o principal fator em análise. Nesta pesquisa, ao se avaliar o potencial cariostático de materiais restauradores em superficie dentinária radicular, consideraram-se apenas os aspectos físico-químicos da formação e do desenvolvimento da lesão cariosa. Os aspectos microbiológicos e os relacionados ao paciente - higienização bucal, dieta, fluxo salivar, obtenção de fluoretos por outras fontes que não o material res- taurador, situação sócio-econômica - embora de extrema relevância, não foram considerados. Dessa forma, os resultados obtidos não se aplicam diretamente à prática clínica. Entretanto, são indispensáveis para, juntamente com outros estudos afins, predizer o desempenho clínico desses materiais.

Sugere-se, então, para pacientes de alto risco/atividade de cárie a indicação do cimento de ionômero de vidro convencional, em dentes onde não haja envolvimento de ordem estética e/ou mecânica. Em situações com comprometimento estético, em que o risco de cárie possa ser controlado, as resinas compostas são indicadas. Outros fatores também devem ser considerados, tais como a relação custo/benefício e as condições técnicas para a aplicação dos materiais, uma vez que o cimento de ionômero de vidro convencional pode ser utilizado na carência de equipamentos odontológicos sofisticados $^{13}$.

\section{CONCLUSÕES}

Dentro das condições aplicadas para a realização deste estudo, foi possível concluir que o cimento de ionômero de vidro convencional (Chelon-Fil) demonstrou um maior potencial cariostático quando comparado à resina composta (Z100) associada a um adesivo hidrófilo (SingleBond), em dentina radicular.

HARA, A. T.; MAGALHÃES, C. S. de; RODRIGUES Jr., A. L.; SERRA, M. C. Caries inhibition around adhesive restorations in roots: in vitro study. Pesq Odont Bras, v. 14, n. 2, p. 113-118, abr./jun. 2000.

Dental materials that release fluoride have been shown to be effective in caries inhibition around restorations. Adhesive materials would also be effective in caries inhibition by sealing and protecting cavity margins from acidic demineralization. This in vitro study tested the hypothesis that composite restorations with a dentin adhesive system have a caries preventive effect similar to that of an adhesive material with fluoride - glass-ionomer cement - on root surfaces. Twenty roots from extracted sound third molars were embedded in polystyrene resin and ground flat. Standardized cavities were prepared in leveled root surfaces and randomly restored with (a) Chelon-Fil (Espe) or (b) $\mathrm{Z} 100 /$ SingleBond (3M). Baseline indentations were measured at 100, 200 and $300 \mu \mathrm{m}$ from the occlusal margins of each restoration and the surface microhardness values were obtained using a Knoop diamond indenter. A $2.0 \mathrm{~mm}$ wide margin around the restorations was submitted to a $\mathrm{pH}$-cycling model, at $37^{\circ} \mathrm{C}$. After that, surface microhardness was measured again, as it was before. The differences between baseline and final surface microhardness were considered for statistical analysis. The median values of differences were (a): $-3.8 ;-0.3 ;-1.0$; and (b): $3.3 ; 2.5 ; 1.7$, for the distances of 100, 200 and $300 \mu \mathrm{m}$, respectively. The Kruskal-Wallis test did not show statistically significant difference between 100, 200 and $300 \mu \mathrm{m}$ distances in each tested group. There was no difference between the studied materials at the distances of 200 and $300 \mu \mathrm{m}$. Chelon-Fil was statistically different from Z100/SingleBond, at $100 \mu \mathrm{m}(\mathrm{p}<0.05)$. Under the studied conditions, the glass-ionomer cement had a higher caries preventive effect than the composite/dentin adhesive restorations.

UNITERMS: Root caries; Cariostatic agents; Dental materials. 
HARA, A. T.; MAGALHÃES, C. S. de; RODRIGUES Jr., A. L.; SERRA, M. C. Efeito cariostático de restaurações adesivas em superficies radiculares: estudo in vitro. Pesq Odont Bras, v. 14, n. 2, p. 113-118, abr./jun. 2000.

\section{REFERÊNCIAS BIBLIOGRÁFICAS}

1. CASTELNUOVO, J.; TJAN, A. H. L.; LIU, P. Microleakage of multi-step and simplified-step bonding systems. Am J Dent, v. 9, n. 6, p. 245-248, Dec. 1996.

2. CREANOR, S. L.; CARRUTHERS, L. M. C.; SAUNDERS, W. P.; STRANG, R.; FOYE, R. H. Fluoride uptake and release characteristics of glass-ionomer cements. Caries Res, v. 28, n. 5, p. 322-328, Sept./Oct.

3. DERAND, T.; JOHANSSON, B. Experimental secondary caries around restorations in roots. Caries Res, v. 18, n. 6, p. 548-554, Nov./Dec. 1984.

4. DIONYSOPOULOS, P.; KOTSANOS, N.; PAPADOGIANNIS, Y.; KONSTANTINIDIS, A. Artificial secondary caries around two new F-containing restoratives. Oper Dent, v. 23, n. 2, p. 81-86, Mar./Apr. 1998.

5. DUNNE, S. M.; GOOLNIK, J. S.; MILLAR, B. J.; SEDDON, R. P. Caries inhibition by a resin-modified and a conventional glass ionomer cement, in vitro. J Dent, v. 24, n. 1-2, p. 91-94, Jan./Mar. 1996.

6. ERICKSON, R. L. Root surface treatment with glass ionomers and resin composites. Am J Dent, v. 7, n. 5, p. 279-285, Oct. 1994.

7. FEATHERSTONE, J. D. B. Fluoride, remineralization and root caries. Am J Dent, v. 7, n. 5, p. 271-274, Oct. 1994.

8. FEATHERSTONE, J. D. B.; O'REILLY, M. M.; SHARIATI, M.; BRUGLER, S. Enhancement of remineralization in vitro and in vivo. In: LEACH, S. A. Factors relating to demineralization and remineralization of the teeth. Oxford : IRL, 1986. p. 23-24.

9. FEJERSKOV, O. Recent advancements in the treatment of root surface caries. Int Dent J, v. 44, n. 2, p. 139-144, Apr. 1994.

10. FEJERSKOV, O.; NYVAD, B. Dental caries in aging individuals. In: HOLM-PEDERSEN, P.; LÖE, H. Textbook of geriatric dentistry. 2. ed. Copenhagen : Munksgaard. 1996. 584 p.

11. FORSTEN, L. Short and long-term fluoride release from glass ionomers and other fluoride-containing filling materials in vitro. Scand J Dent Res, v. 98, n. 2, p. 179-185, Apr. 1990 .

12. FOYE, R. H.; GILMOUR, W. H. The effect of a resin-modified glass ionomer restorative material on artificially demineralised dentine caries in vitro. J Dent, v. 26, n. 5-6, p. 527-531, July/Aug. 1998.

13. FRENCKEN, J. E.; MAKONI, F.; SITHOLE, W. D.; HACKENITZ, E. Three-year survival of one-surface ART restorations and glass-ionomer sealants in a school oral health programme in Zimbabwe. Caries Res, v. 32, n. 2, p. 119-126, Mar./Apr. 1998.

14. GILMOUR, A. S. M.; EDMUNDS, D. H.; NEWCOMBE, R. G. Prevalence and depth of artificial caries-like lesions adjacent to cavities prepared in roots and restored with a glass-ionomer or a dentin-bonded composite material. $\mathbf{J}$ Dent Res, v. 76, n. 12, p. 1854-1861, Dec. 1997.

15. KIDD, E. A. M.; TOFFENETTI, F.; MJÖR, I. A. Secondary caries. Int Dent J, v. 42, n. 3, p. 127-138, June 1992.

16. MILLAR, J. B.; ABIDEN, F.; NICHOLSON, J. W. In vitro caries inhibition by polyacid-modified composite resins (compomers). J Dent, v. 26, n. 2, p. 133-136, Mar. 1998.

17. MITRA, S. B. In vitro fluoride release from a light-cured glass-ionomer liner/base. J Dent Res, v. 70, n. 1, p. 75-78, Jan. 1991.

18. MJÖR, I. A.; JOKSTAD, A. Five-year study of class II restorations in permanent teeth using amalgam, glass polyalkenoate (ionomer) cermet and resin-based composite materials. J Dent, v. 21, n. 6, p. 338-343, Dec. 1993.

19. NAGAMINE, M.; ITOTA, T.; TORII, Y.; IRIE, M.; STANINEC, M.; INOUE, K. Effect of resin-modified glass-ionomer cements on secondary caries. Am J Dent, v. 10, n. 4, p. 173-178, Aug. 1997.

20. NYVAD, B.; FEJERSKOV, O. Active root surface caries converted into inactive caries as a response to oral hygiene. Scand J Dent Res, v. 94, n. 3, p. 281-284, Jun. 1986.

21. NYVAD, B.; TEN CATE, J. M.; FEJERSKOV, O. Arrest of root surface caries in situ. J Dent Res, v. 76, n. 12, p. 1845-1853, Dec. 1997.

22. ÖSTLUND, J.; MÖLLER, K.; KOCH, G. Amalgam, composite resin and glass ionomer cement in class II restorations in primary molars - a three year clinical evaluation. Swed Dent J, v. 16, n. 3, p. 81-86, 1992.

23. PEREIRA, P. N. R.; INOKOSHI, S.; TAGAMI, J. In vitro secondary caries inhibition around fluoride releasing materials. J Dent, v. 26, n. 5-6, p. 505-510, July/Aug. 1998.

24. RANDALL, R. C.; WILSON, N. H. F. Glass-ionomer restoratives: a systematic review of a secondary caries treatment effect. J Dent Res, v. 78, n. 2, p. 628-637, Feb. 1999.

25. RODRIGUES, J. A.; MAGAlHÃES, C. S.; SERRA, M. C.; RODRIGUES Jr., A. L. In vitro microleakage of glass-ionomer composite resin hybrid materials. Oper Dent, v. 24, n. 2, p. 89-95, Mar./Apr. 1999.

26. SERRA, M. C.; CURY, J. A. The in vitro effect of glass ionomer cement restoration on enamel subjected to demineralization and remineralization model. Quintessence Int, v. 23, n. 2, p. 143-147, Feb. 1992.

27. SERRA, M. C.; RODRIGUES JÚNIOR, A. L. Potencial cariostático de materiais restauradores contendo flúor. Rev Assoc Paul Cir Dent, v. 52, n. 5, p. 359-364, set./out. 1998.

28. SETTEMBRINI, L.; GULTZ, J. P.; SCHERER, W.; KAIM, J. A single-component bonding microleakage study. Gen Dent, v. 45, n. 4, p. 341-343, July/Aug. 1997.

29. TAM, L. E.; CHAN, G. P.-L.; YIM, D. In vitro caries inhibition effects by conventional and resin-modified glass-ionomer restorations. Oper Dent, v. 22, n. 1, p. 4-14, Jan./Feb. 1997.

30. TEN CATE, J. M. In vitro studies on the effects of fluoride on de- and remineralization. J Dent Res, v. 69 (SI), p. 614-619, Feb. 1990.

31. THYLSTRUP, A. How should we manage initial and secondary caries? Quintessence Int, v. 29, n. 9, p. 594-598, Sept. 1998.

32. WEFEL, J. S. Root caries histopathology and chemistry. Am J Dent, v. 7, n. 5, p. 261-265, Oct. 1994.

33. WILSON, A. D.; KENT, B. E. A new translucent cement for dentistry. The glass-ionomer cement. Br Dent J, v. 132, n. 4, p. 133-135, Feb. 1972
Recebido para publicação em 19/01/00

Enviado para reformulação em 15/02/00

Aceito para publicação em 30/03/00 